\title{
A comparison of method-of-adjustment and forced-choice procedures in frequency discrimination
}

\author{
CRAIG C. WIER, WALT JESTEADT, and DAVID M. GREEN \\ Laboratory of Psychophysics, Harvard University, Cambridge, Massachusetts 02138
}

\begin{abstract}
Reported estimates of the frequency difference limen (DL) for tones show considerable variability. To determine the extent that the differences are dependent on psychophysical method, three estimates of the DL at $1,000 \mathrm{~Hz}$ were obtained from the same subjects for each of three psychophysical procedures. The three estimates were: (1) the standard deviation of final settings in a method of adjustment, (2) the average of several reversals in an adaptive two-interval forced-choice procedure, and (3) the $76 \%$-correct point in a two-interval forced-choice procedure using constant stimuli. The two forced-choice procedures yielded very similar DLs. The adjustment procedure yielded significantly smaller estimates. Possible reasons for the different values produced by adjustment procedures and the nature of the underlying decision process are discussed.
\end{abstract}

The absolute size of the frequency difference limen (DL) has long been of interest to sensory psychologists. Estimates of the frequency DL show considerable variability across experiments as several investigators have observed (Boring, 1940; Luce \& Green, 1974; Rosenblith \& Stevens, 1953). Figure 1 shows some representative data on frequency discrimination.

This variability may result from the wide range of stimulus configurations and psychophysical methods used to measure frequency discrimination. As a preliminary to an extensive study of the frequency DL at several different frequencies and intensities, we measured the frequency DL using three different psychophysical methods. We present these data here because these measurements provide further evidence about the relations among methods which may apply to other sensory dimensions as well.

The most familiar data on the frequency DL are undoubtedly those of Shower and Biddulph (1931). These data represent performance with frequencymodulated tones (FM), and provide an upper boundary for the data shown in Figure 1. Several investigators. notably Harris (1952) and Stevens (1954), have pointed out that frequency DLs for FM tones are consistently larger than DLs obtained with pulsed sinusoids, particularly at low frequencies. More recently, Jesteadt and Sims (1975) compared performance with these two stimulus configurations for the same group of subjects and found the DLs for FM tones to be larger than the DLs for pulsed sinusoids. Comparison of the results for individual subjects showed little or no correlation in performance on the two tasks. A considerable portion of the range

This research was supported by grants from the National Institutes of Health. Portions of this article were presented at the meeting of the Acoustical Society of America, Austin, Texas, 1975. The authors wish to thank R. Mayberry and B. C. J. Moore for their comments on an earlier draft.

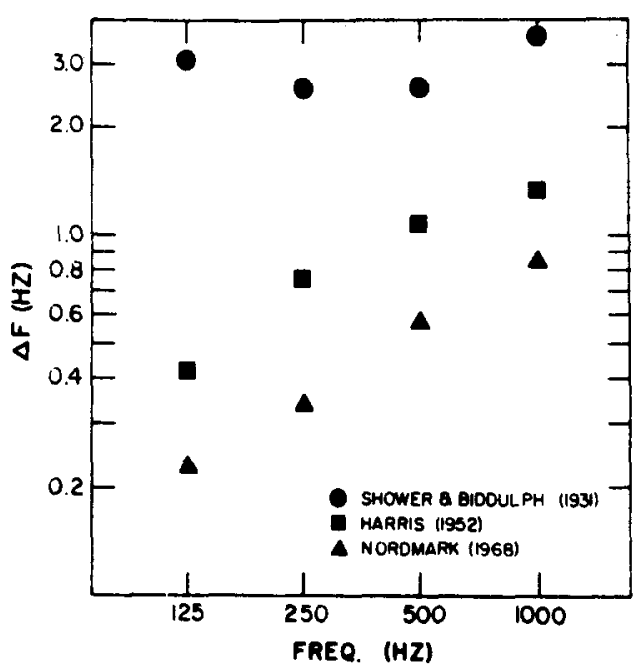

Figure 1. Estimates of the frequency DL.

shown in Figure 1 can be accounted for by this difference in stimulus configuration; both the lower functions are based on pulsed sinusoids.

Although both Nordmark's (1968) and Harris (1952) data were obtained using pulsed sinusoids, they differ considerably. Apart from the variables of training and experience that always enter in to comparisons across laboratories and subjects, one difference between these experiments is the use of different psychophysical procedures. Harris used a two-interval forced-choice (2IFC) constant-stimulus procedure. The DL was defined as the stimulus difference that produced $75 \%$-correct responses. Nordmark used an adjustment procedure in which the subjects controlled the frequency of one of two alternating tones. The subjects were instructed to set the two tones equal to one another, and no time limit was imposed. The DL was defined as the standard deviation of a number of such adjustments. 
In psychophysics, the use of the theory of signal detection has led to the development of models of sensory and decision processes that make explicit, empirically testable predictions about the relations among different procedures. Paradigm comparisons have been made for frequency-discrimination performance among most of the standard forced-choice procedures: yes-no, 2IFC, samedifferent (Jesteadt \& Bilger, 1969; Jesteadt \& Sims, 1975). The data from these comparisons support the basic assumptions of signal detection theory concerning the decision process, but suggest that an additional factor, such as memory, is also operating in some procedures (Durlach \& Braida, 1969; Tanner, 1961).

Attempts to relate measures of discrimination across psychophysical methods have generally ignored the adjustment procedures. Cardozo (1965) provides a limited quantitative description of the adjustment procedure without consideration of possible decision processes. He predicts that the standard deviation of the adjustments will be nearly a factor of 2 smaller than the stimulus difference $(\Delta F)$ required for $75 \%$ correct in 2IFC.

A number of early investigators compared adjustment procedures with various versions of the method of constant-stimulus differences (see Kellogg, 1929, for a review). Despite differences in stimuli and procedures, forced-choice methods typically produced DLs larger than those obtained with adjustment procedures, frequently larger by a factor of about 2 . Probably the most ambitious and pertinent of these early experimental attacks on the problem was that of Kellogg (1929). He used both adjustment and constant-stimulus-difference methods to obtain DLs for both light and sound intensities. He found that, in 106 out of 120 cases, the standard deviation obtained with the adjustment procedure was smaller than the corresponding standard deviation estimate obtained with the constant-stimulus-difference method.

The data in Figure 1 representing recent estimates of frequency DLs also show a smaller DL for the adjustment procedure - smaller by about a factor of 2 , as observed in the historical literature and consistent with Cardozo's suggestion. Thus, it appears that the differences in the estimates of the DL obtained by the adjustment and forced-choice procedures may account for a significant portion of the variability which remains in the frequency-DL data after the influence of stimulus configuration is taken into account. The variability in reported estimates of the frequency $\mathrm{DL}$ suggests that comparisons among methods should be made using the same subjects. In the following experiment, we obtained estimates of the frequency DL for the same subjects using both an adjustment and a constant-stimulus-difference forced-choice procedure. We also used an adaptive-2IFC procedure, with characteristics common to both adjustment and forced-choice procedures (see Levitt, 1971).

\section{METHOD}

\section{Subjects}

Six subjects were paid to participate in the experiment. Five of the six subjects had had no previous laboratory listening experience; the sixth subject had had several months' experience listening in forced-choice procedures. The musical experience of the subjects ranged from that of one subject who was a member of the local opera's chorus to no formal musical training at all.

\section{Apparatus}

In the forced-choice procedures, stimulus presentation and response record keeping were under computer control. The stimuli were generated by Wavetek (Model 116) oscillators. Subjects listened monaurally with headphones in individual sound-treated rooms (IAC-1200). In the adjustment procedure, listening conditions were similar, except the frequency of the variable stimulus was under the control of the subject who turned a 10-turn potentiometer mounted in his listening booth which varied the voltage input to the voltage-controlled oscillators.

\section{Procedure}

Time lines for the psychophysical procedures are shown in Figure 2. In all of the procedures, the frequency of the standard was $1,000 \mathrm{~Hz}$ and all stimuli were presented at $70 \mathrm{~dB}$ SPL. The temporal relations of the stimuli were held constant across procedures. Tones were always $500 \mathrm{msec}$ in duration with a 500 -msec silent interval between the observation intervals in the forced-choice procedures and between each member of a stimulus pair in the adjustment procedure.

In the forced-choice procedures, subjects were instructed to indicate the interval which contained the higher frequency tone. They responded, received feedback, and the next trial began. For the constant-stimulus condition, subjects listened to three blocks of 100 trials each at each value of $\Delta F$, thereby generating three-point psychometric functions based on 900 trials. The adaptive procedure was a 2-down, 1-up procedure for which the mean of the turn-around points is an estimate of the $71 \%$-correct point on the psychometric function (Levitt, 1971). The estimates from 10 100 -trial blocks were averaged.

In the adjustment procedure, unlike the forced-choice procedures, the order of the standard and standard $\pm \Delta F$ was fixed. The frequency of the first tone was always $1,000 \mathrm{~Hz}$, and the frequency of the second tone was under the control of the subject. A 1 -sec interval separated stimulus pairs to approximate the response-feedback interval in the forced-choice procedures.

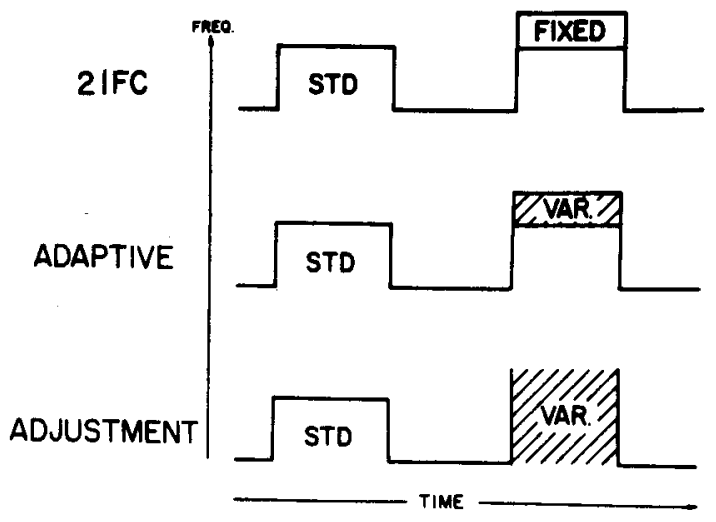

Figure 2. Time lines for the paychophysical procedures. 
Subjects were instructed to adjust the frequency of the second tone to be equal to that of the first. The initial frequency of the comparison tone was varied at the start of each run to be either above or below the standard in order to avoid bias and knob-position cues. No time limit was imposed. Subjects typically listened to about 40 stimulus pairs while manipulating the frequency of the second tone before reporting a match. The frequency of the variable tone was then measured and the procedure started over. Each subject made 15 such matches. The six subjects were divided into three pairs; each pair encountered the three psychophysical procedures in a different order.

\section{RESULTS}

Figure 3 shows the results for five of the subjects for each of the procedures. ${ }^{1}$ The filled circles represent the mean performance for the adaptive procedure, and the vertical bars are one standard deviation about the mean. The open circles are the estimates from the constant-stimulus psychometric functions and represent the $\Delta F$ necessary for $71 \%$-correct responses. ${ }^{2}$ It is clear that the adaptive procedure produces a reasonable and unbiased estimate of the performance measured by the more traditional 2IFC procedure. A two-tailed $t$ test for related measures indicates that the estimates from these two procedures are not significantly different. A further comparison is provided by plotting the individual psychometric functions for both forced-choice procedures. Complete trial-by-trial records of stimulus-response relations in the adaptive procedure were available for three of the subjects. ${ }^{3}$ Figure 4 shows the psychometric functions for the adaptive procedure plotted along with the constant-stimulus functions from the same subjects. ${ }^{4}$ Again, the agreement between the two procedures is quite good.

The adjustment data shown in Figure 3 are consistently lower than the data from the forced-choice procedures. One-tailed $t$ tests for related measures indicate that the method-ofadjustment data are significantly different from both the forced-choice procedures ( $p<.05$ for both tests).

The mean DLs for each procedure are shown for five subjects in Table 1 . The mean DL from the constant-stimulus forced-choice procedure when measured at $\mathrm{d}^{\prime}=1.0$ ( $76 \%$ correct) is a factor of 2 larger than the DL from the adjustment procedure.

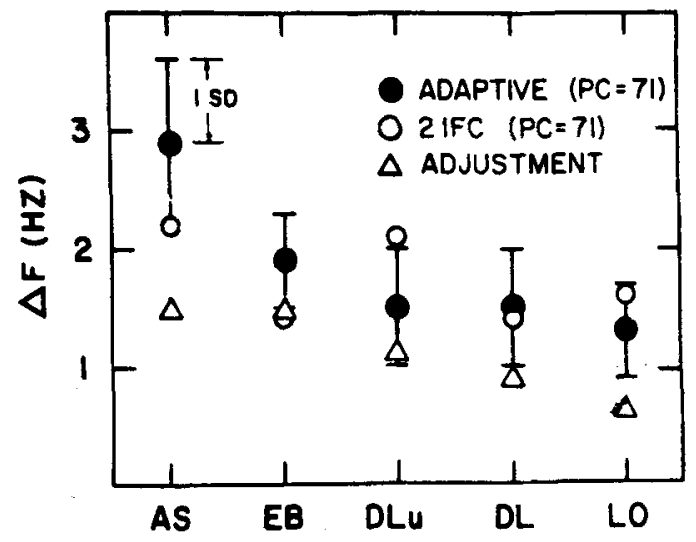

Figure 3. Individual subjects' mean performance for each psychophysical procedure.

This is the same size factor shown in Figure 1 and suggested by both the historical literature and Cardozo. In the present case, the difference between procedures remains when the comparison is within subjects. Therefore, it cannot be attributed to differences in frequency discrimination ability or training. The two middle columns in Table 1 show the $\Delta F s$ interpolated from the same points on the psychometric functions. The two forced-choice procedures yield the same mean estimates.

\section{DISCUSSION}

In addition to the effects of stimulus configuration, mentioned earlier, the results of this experiment demonstrate that the use of different psychophysical procedures also influences the estimate of the frequency DL. Estimates obtained from individual subjects with the adjustment procedure are about a factor of 2 smaller, on the average, than the estimates obtained with forced choice. Using the adjustment procedure, even our relatively untrained listeners (total of $\sim 6 \mathrm{~h}$ listening, including the experiment) produced DLs for $1,000-\mathrm{Hz}$ pure tones that were smaller than any we know of in the literature for forced-choice procedures.

The adjustment procedure involves a number of variables which undoubtedly influence the results but
Figure 4. Psychometric functions for the forced-choice procedures. Filled circles, 2IFC; open circles, adaptive.

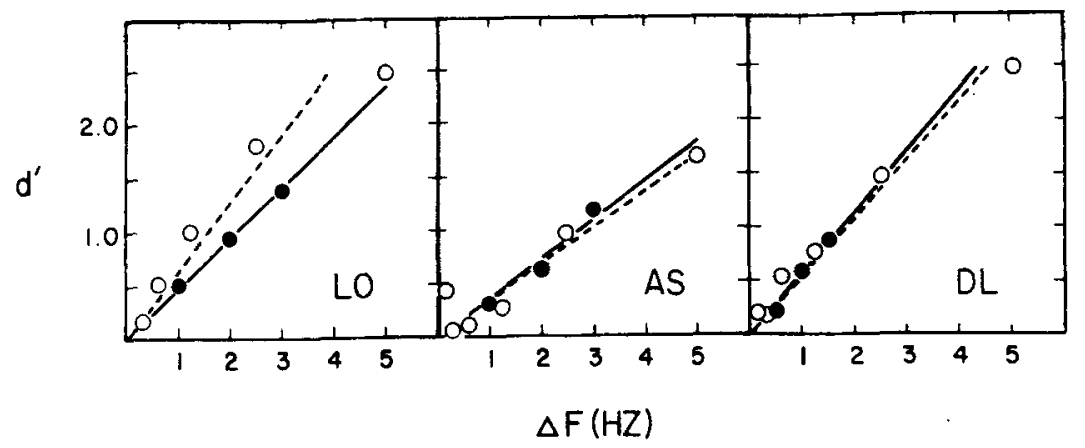


Table 1

Estimates of the Frequency DL (Hz)

\begin{tabular}{ccccc}
\hline Subjects & $\begin{array}{c}\text { 2IFC } \\
\left(\mathrm{d}^{\prime}=1.0\right)\end{array}$ & $\begin{array}{c}\text { 2IFC } \\
(\mathrm{PC}=71)\end{array}$ & $\begin{array}{c}\text { Adaptive } \\
(\mathrm{PC}=71)\end{array}$ & $\begin{array}{c}\text { Adjust- } \\
\text { ment } \\
(\mathrm{SD})\end{array}$ \\
\hline A.S. & 2.8 & 2.2 & 2.9 & 1.5 \\
E.B. & 1.8 & 1.4 & 1.9 & 1.5 \\
D.Lu. & 2.7 & 2.1 & 1.5 & 1.1 \\
D.L. & 1.8 & 1.4 & 1.5 & .9 \\
L.O. & 2.1 & 1.6 & 1.3 & .6 \\
Mean & 2.2 & 1.8 & 1.8 & 1.1 \\
\hline
\end{tabular}

which are difficult, if not impossible, for the experimenter to control. Some of these factors are not sensory in nature, e.g., the coarseness of the knob the subject adjusts which effectively limits the minimum step size, the number of stimulus presentations sampled, and the subjects' strategy for terminating listening.

Cardozo describes the adjustment procedure as a random walk as the average stimulus difference approaches zero. The relation between the distribution of stimulus values and the psychometric function is determined by the average step size the subject uses in making adjustments. The relation between the standard deviation of the distribution of stimulus values following the final adjustment and the $75 \%$ point on the psychometric function is

$$
\sigma=(\xi \delta)^{1 / 2}
$$

where $\sigma$ is the standard deviation of the adjustments, $\xi$ is the average step size, and $\delta$ is the stimulus difference corresponding to $75 \%$ correct in a 2 IFC procedure. Cardozo found that his better subjects typically selected a value of $\xi=1 / 3 \delta$, resulting in values of $\sigma$ nearly a factor of 2 smaller than $\delta$. No assumptions are made, and no predictions are possible, concerning the subjects' rules for terminating the adjustment process. It should be noted here that Cardozo does not claim that his analysis constitutes a complete model of the adjustment procedure.

When the method of adjustment is used to measure the DLs, the subjects' usual task is to set a variable stimulus as nearly equal to a fixed stimulus as possible. Normally, the subject is free to sample any stimulus value and listen to as many stimulus presentations as he wishes before making each adjustment. Data for forced-choice procedures suggest that multiple observations permit more accurate adjustments (Swets, Shipley, McKey, \& Green, 1959). While there is generally no time pressure or reward for speed, it is likely that the subject attempts to reach a final adjustment in a minimum amount of time. We assume that in a discrete-trial task such as that employed here, the subject samples the fixed stimulus and the current value of the variable stimulus and makes a high-same-low decision. If the decision is high or low, the subject makes the appropriate adjustment and samples a new value of the variable stimulus. If the decision is same, the subject makes no adjustment and samples the current value again to gather additional information. If the subject is unable to reach a decision of high or low after several presentations, we assume that the current value of the variable stimulus is accepted as a final value. As a minor variation on this approach, the subject might attempt to establish the upper and lower boundaries of the uncertainty region and then select a middle value as a final setting. Some references recommend this variant (see Guilford, 1936).

The preceding description is essentially the application of sequential decision making (Wald, 1947) to the adjustment procedure. This decisionmaking analysis has been applied in other areas of psychophysics (Laming, 1968; Stone, 1960; Swets \& Birdsall, 1967; Swets \& Green, 1961). Without going into more detail, it should be clear that both step size and the number of repeated stimulus presentations influence the DL measured in the method of adjustment.

As yet, no one has presented an analysis of how these two variables can be used to estimate the sensitivity of the subject or the decision criteria. Until such an analysis is presented and varified, it will be difficult to relate adjustment data and forced-choice data.

The choice of an alternative psychophysical procedure, such as the adaptive procedure used in the present study, avoids many of the problems associated with adjustment procedures. The relations between adaptive procedures and constant-stimulus forcedchoice procedures have been described in detail (Levitt, 1971; Taylor \& Creelman, 1967). The validity of such descriptions is demonstrated by the data in Figures 3 and 4 . Adaptive procedures retain a principal advantage of the adjustment procedures. They do not require the experimenter to know the range of stimulus values that will describe individual psychometric functions.

\section{REFERENCES}

Boring, E. G. The size of the differential limen for pitch. American Journal of Psychology, 1940, 53. 450-455.

CARDozo, G. L. Adjusting the method of adjustment: SD vs DL. Journal of the Acoustical Society of A merica. 1965, 37, 786-792.

Durlach, N. I., \& Braida, L. D. Intensity perception. I. Preliminary theory of intensity resolution. Journal of the Acoustical Society of America, 1969, 46, 372-383.

GuILford, J. P. Psychometric methods. New York: McGraw-Hill, 1936. 
HARRIS, J. D. Pitch discrimination. Journal of the Acoustical Society of America, 1952, 24, 750-755.

JESTEADT, W., \& Bilger, R. C. Frequency discrimination near masked threshold. Perception \& Psychophysics, 199, 6, $405-408$.

JESTEADT, W., \& Sims, S. L. Decision processes in frequency discrimination. Journal of the Acoustical Society of America, 1975, 57. 1161-1168.

Kellogg, W. N. An experimental comparison of psychophysical methods. Archives of Psychology, 1929, No. 106.

LAMING, D. R. J. Information theory of choice-reaction times. London: Academic Press, 1968.

LEVITT, H. Transformed up-down methods in psychoacoustics. Journal of the Acoustical Society of America, 1971, 49, 467-477.

LuCE, R. D., \& GREEN, D. M. Neural coding and psychophysical discrimination data. Journal of the Acoustical Society of America, 1974, 56, 1554-1564.

Nordmark, J. O. Mechanisms of frequency discrimination. Journal of the Acoustical Society of America, 1968, 44. 1533-1540.

Rosenblith, W. A., \& Stevens, K. N. On the DL for frequency. Journal of the Acoustical Society of America, 1953, 25, 980-985.

ShOWER, E. G., \& Biddulph, R. Differential pitch sensitivity of the ear. Journal of the Acoustical Society of America, $1931,3,275-287$

Stevens, S. S. Pitch discrimination. mels, and Kock's contention. Journal of the Acoustical Society of America, 1954, 26, 1075-1077.

Stone, M. Models for choice reaction time. Psychometrika, 1960, 25, 251-260.

Swets, J. A., \& Birdsall, T. G. Deferred decision in human signal detection: A preliminary experiment. Perception \& Psychophysics, 1967, 2, 15-28.

Swets, J. A., \& Green, D. M. Sequential observations by human observers of signals in noise. In C. Cherry (Ed.), Information theory. London: Butterworths, 1961.
Swets, J. A., Shipley, E. F., McKey, M. J., \& Green, D. M. Multiple observations of signals in noise. Journal of the Acoustical Society of America, 1959, 31, 514-521.

TANmer, W. P., JR. Physiological implications of psychophysical data. Annals of the New York Academy of Sciences, 1961, 89, $752-765$.

TAYlor, M. M., \& Creelman, C. D. PEST: Efficient estimates on probability functions. Journal of the Acoustical Society of America, 1967, 41, 782-787.

WALD, A. Sequential analysis. New York: Wiley, 1947.

\section{NOTES}

1. The one experienced subject in the group expressed considerable confusion in the adjustment task. He was accustomed to listening in forced-choice procedures and produced the lowest DLs in both forced-choice tasks. In the adjustment procedure, he found the lack of immediate feedback very distressing. Although we assume we could have spent some time cajoling him to produce performance in the adjustment procedure similar to that produced by the other subjects, the constraints of time and the intentions of the experiment led us to drop him from the final analysis.

2. The constant-stimulus forced-choice procedure data were fit with a least squares fit through the origin in $d^{\prime}-b y-\Delta F$ space. Percent correct estimates were then derived from the $d^{\prime}$ estimates.

3. Trial-by-trial data for the remaining subjects was lost due to tape errors.

4. The adaptive-procedure data were fit with a least squares procedure in which squared deviations were weighted according to the number of trials contributing to each point.

(Received for publication September 19, 1975; accepted October 15, 1975. ) 